SPECIAL ISSUE ON STRUCTURE IN GLASSY AND JAMMED SYSTEMS • OPEN ACCESS

The potential energy landscape for crystallisation of a Lennard-Jones fluid

To cite this article: Vanessa K de Souza and David J Wales J. Stat. Mech. (2016) 074001

View the article online for updates and enhancements.
Related content

Computer simulations of glasses: the potential energy landscape

Zamaan Raza, Björn Alling and Igor A Abrikosov

Energy landscapes and properties of biomolecules David J Wales

Challenges in molecular simulation of homogeneous ice nucleation Andrey V Brukhno, Jamshed Anwar, Ruslan Davidchack et al.

\section{Recent citations}

Optimal Alignment of Structures for Finite and Periodic Systems

Matthew Griffiths et al 


\title{
The potential energy landscape for crystallisation of a Lennard-Jones fluid
}

\author{
Vanessa K de Souza and David J Wales \\ University Chemical Laboratories, Lensfield Road, Cambridge CB2 1EW, UK \\ E-mail: dw34@cam.ac.uk
}

Received 1 February 2016

Accepted for publication 25 April 2016

Published 1 July 2016

Online at stacks.iop.org/JSTAT/2016/074001

doi:10.1088/1742-5468/2016/07/074001

\begin{abstract}
Crystallisation pathways are explored by direct analysis of the potential energy landscape for a system of Lennard-Jones particles with periodic boundary conditions. A database of minima and transition states linking liquid and crystalline states is constructed using discrete path sampling and the entire potential energy landscape from liquid to crystal is visualised. We demonstrate that there is a strong negative correlation between the number of atoms in the largest crystalline cluster and the potential energy. In common with previous results we find a strong bias towards the growth of FCC rather than HCP clusters, despite a very small potential energy difference. We characterise three types of perfect crystals with very similar energies: pure FCC, pure HCP, and combinations of FCC and HCP layers. There are also many slightly defective crystalline structures. The effect of the simulation box is analysed for a supercell containing 864 atoms. There are low barriers between some of the different crystalline structures via pathways involving sliding layers, and many different defective structures with FCC layers stacked at an angle to the periodic box. Finally, we compare a binary Lennard-Jones system and visualise the potential energy landscape from supercooled liquid to crystal.
\end{abstract}

Keywords: energy landscapes (theory) 


\section{Introduction Contents}

Crystallisation is the formation of an ordered solid from a disordered liquid. The average waiting troduction

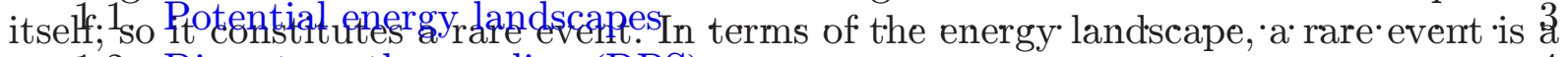

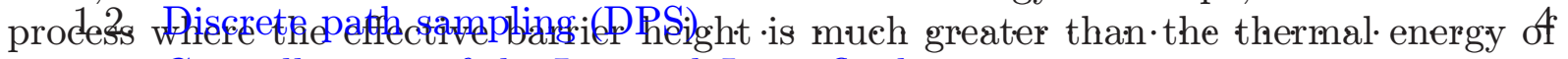

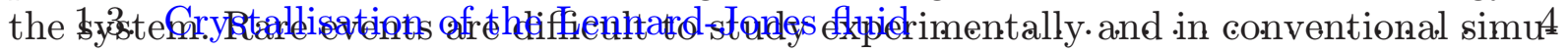
latidn\$. Sysstiemuldetoms the problem is .obtaining sufficient statistics, . while .in experi5

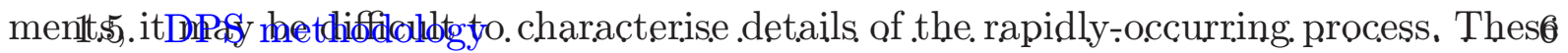
detail.6. ane eimpertata critical noslebs that afe masthrelevant to theories of crystal nucleation. Crystal nuclej form spontaneously in a supersaturated solution, but redissolve rather than grow, Znlesesults size exceeds a critical value. The probability that a critical nucleus form 8

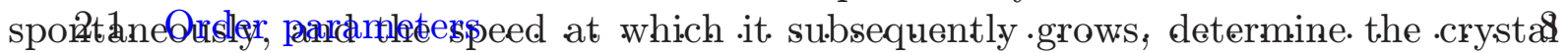
nucleationo watergystatlistatien can be studied experimentally. with colloidal suspeht

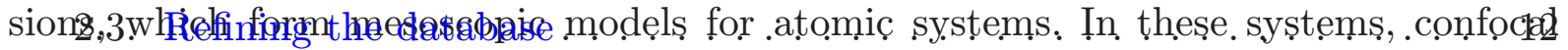

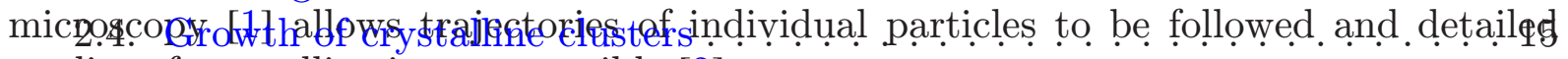
studies. of athstallisation are possible [2].

Aumer fo simation techn 2.6. Binary Lennard-Jones. events. These techniques include parallel replica dynamics [3], umbrella sampling [1], Coaddustiodsmolecular dynamics [5], metadynamics [6, 7], temperature acceler ated dynamics [8], transition path sampling [9-11], forward-flux sampling [12, 132 and adaptive kinetic Monte Carlo [14]. The approach we will use here, discrete path samplifigrendes [15-17], was developed as an analogue of transition path samplir22 coarse-grained in terms of stationary points of the potential energy surface, which are determined by geometry optimisation techniques. The reasons for applying this technique to a well-studied problem, the crystallisation of the Lennard-Jones fluid, are two-fold. Firstly, we wish to demonstrate the application of the DPS approach to bulk systems. The technique can then be used for systems that do not crytsallise easily and where order parameters are unknown. This development requires the introduction of structural alignment algorithms to identify isomers and structural similarities in periodic boundary conditions. Secondly, DPS gives us the opportunity to visualise the full potential energy landscape from liquid to crystal and to examine each region in detail. In the liquid region, we examine stacking preferences during nucelation and in the crystal region, we study the effect of periodic boundary conditions on the crystal structures and the pathways between them.

\subsection{Potential energy landscapes}

A potential energy surface (PES), or landscape (PEL), represents the potential energy of a given system as a function of all the relevant atomic or molecular coordinates [18]. Usually the most interesting parts of a potential energy surface are the stationary points, where all the components of the gradient vector are zero. These stationary points include minima and transition states. At a local minimum any small change in the internal coordinates results in an increase in energy. The minimum with lowest 
energy is known as the global minimum. Equilibrium thermodynamic properties depend only on the relative potential energies of the local minima on the PES and the volumes of configuration space associated with them. However, relating the potential energy surface to dynamics requires information about the connections between different minima. Geometrically, the lowest-energy pathway between two directly connected minima proceeds via a transition state and corresponding steepest-descent pathways. Here we employ the Murrell and Laidler definition of a transition state, namely a stationary point with a single negative Hessian eigenvalue [19].

For small clusters, it is possible to perform an exhaustive search of the PES for stationary points [20]. However, the number of minima for a system of $N$ atoms increases exponentially $[21,22]$ with $N$, making exhaustive searches of the PES for large systems impossible. Instead, we must consider incomplete databases of minima and transition states that are representative of the region of the PES explored by the system under experimental conditions.

The thermodynamics of the system are determined by the entire PES. However, the superposition approach [22-26] may be applied to obtain properties from a representative sample of local minima. Similarly, global kinetics can be analysed in terms of transitions between individual minima using statistical rate theories for the minimumto-minimum rate constants [18].

The structure and dynamics of atomic and molecular clusters, the folding of proteins, and the phenomenology of glasses, can all be examined in terms of the underlying potential energy landscape [18]. Global optimisation to determine the lowest minimum is a well established technique [27]. Analysing the energy landscape is also an important tool for crystal structure prediction, in finding both the lowest energy crystal and any competing low-energy structures. In the present work, we will explore, not just the lowenergy crystalline region of the landscape, but also pathways to this region from the liquid state. Studying the potential energy landscape allows us to focus more directly on structural properties and on the connections between different morphologies.

\subsection{Discrete path sampling (DPS)}

A discrete path is defined as a connected sequence of local minima and the intervening transition states that connect them geometrically, as opposed to the dynamical trajectories sampled in transition path sampling. DPS and related methods [28-31] employ geometry optimisation techniques to construct a database of connected sequences of stationary points that link two chosen end point states (or regions) in configuration space. Pathways between two different states are examined by looking directly at the potential energy landscape. The DPS method has previously been employed to study isomerisation in model atomic and molecular clusters $[15,16]$, and to examine folding [32-35], misfolding [36] and conformational changes [37] in peptides and proteins.

The discrete path sampling approach was designed to find an initial connected path between two endpoints and then to systematically generate the most kinetically relevant discrete paths from this initial path, enlarging the database of connected stationary points. A discrete path between two distant minima can be constructed iteratively. First, a double-ended transition state (TS) search is undertaken, characterising transition states on the pathway between the two minima $[15,16]$. The resulting TS guesses 
The potential energy landscape for crystallisation of a Lennard-Jones fluid

are tightly converged using single-ended geometry optimisation techniques [38, 39]. The two local minima each TS connects are then identified using local minimisation. The transition states and minima are added to a database and the search begins again. The process is continued with new pairs of end-point minima to bridge the gaps until a complete path is formed. We used the missing connection algorithm [40] to choose successive pairs of minima for the connection attempts. This approach uses Dijkstra's algorithm [41] to identify the sequence of minima on the 'shortest path' in terms of missing connections, where a suitable metric is defined to quantify the path 'lengths' [40].

\subsection{Crystallisation of the Lennard-Jones fluid}

Systems of particles interacting via a Lennard-Jones (LJ) potential have been widely studied as models that capture the essential aspects of the underlying physics of crystallisation, while retaining a simple functional form. There is a considerable body of numerical data available and the phase diagram is well known. At low temperature the stable form is a face-centred cubic (FCC) crystal, and there is a metastable hexagonalclose-packed crystal (HCP) with very similar free energy. However, there is also a metastable body-centred-cubic (BCC) structure with higher free energy, which may play a role in nucleation and crystallisation under certain conditions [42, 43]. The equilibrium volume for BCC is larger than for the close-packed HCP and FCC, and this lattice is not stable. However, it was recently found that the formation of a closepacked structure can be prevented if the system is relaxed from a BCC structure with varying volume. A new metastable phase is formed, with a distorted BCC structure, İ̄3d symmetry, and an equilibrium volume only slightly smaller than for BCC [44].

Crystallisation in bulk Lennard-Jones systems has been studied by 'brute-force' molecular dynamics as well as a number of different rare-event methods [45], including umbrella sampling [42, 43, 46, 47], forward-flux sampling [48], and transition interface path sampling $[49,50]$. It has been shown $[42,43,47,49,50]$ that small clusters form with some BCC structure. As the clusters grow, the surfaces retain BCC character, but the cores transform into FCC packing. Otswald's 'step' rule [51] states that the phase closest in free energy to the fluid is formed first, even if this is not the most stable state, in agreement with this observation. It is also possible to interpret the increase in BCC character at the surface as an increase in FCC particles within the core, due to the greater stability of the bulk FCC phase [50]. Polymorph selection during the growth step has also been studied [43], and BCC crystallites were found to be favoured over FCC at high pressure. Raising the temperature, i.e. running simulations at low supercooling, prevents the cross-nucleation of HCP within the FCC crystallites [43].

HCP and FCC stacking are characterised by close-packed layers of triangularly arranged spheres. The two structures have the same density and differ only in the stacking sequence. Other stacking sequences are also possible with combinations of of FCC and HCP layers, and such structures are commonly identified as random hexagonally close-packed ( $\mathrm{rHCP}$ ) structures. Although they have almost the same density, energetic factors such as long-range interactions can lead to a preference for one structure over another, and there are also small entropic differences [52]. In fact, for the Lennard-Jones potential, and even for monodisperse hard-sphere systems, there is a strong preference for FCC stacking during crystal growth, and 
hence in the formation of crystals, despite little energetic or entropic gain. The preference for FCC packing also appears to depend on how the close-packed crystal is generated, suggesting a mechanical instability acting during crystal formation [53]. It has been shown that close-packed structures form with equal probability. However, the FCC structure is mechanically more stable and less often destroyed in the continuing dynamical growth process, as newly arrived atoms impact upon the structure [53].

\subsection{System details}

We consider a supercell containing 864 atoms interacting via the Lennard-Jones potential with a number density of 1.2 in reduced Lennard-Jones units of $\sigma^{-3}$, for a cubic box with periodic boundary conditions. The cutoff for the potential is at $2.5 \sigma$. This periodic box allows the formation of both a perfect face-centred cubic (FCC) crystal and a perfect hexagonal close-packed (HCP) crystal. Simulating at constant volume allows us to study the effect of the periodic box on the crystal structures formed and the pathways between crystal structures. An NPT study of the PEL would be possible but much more computationally intensive, and in the present contribution we focus on the organisation of the potential energy landscape, rather than rates and nucleation barriers.

When examining the PEL, it is desirable to consider a small supercell, as there is an exponential increase in the number of local minima with the number of atoms $[22,21]$. Large systems can be decomposed into weakly interacting subsystems, which can mask information when considering properties [54-56]. However, a study of crystallisation via nucleation requires a system big enough to accommodate the critical nucleus without the risk of forming nuclei that percolate through the periodic box. Previous studies of crystallisation in Lennard-Jones systems suggest a critical nucleus of between 100 and 200 atoms with approximately 30\% undercooling [50, 57]. Hence, our system is big enough to study precritical clusters, but will produce artifacts in terms of growth after the formation of the critical nucleus, as structure can propagate readily across the periodic boundaries. It has been suggested that the most important and detectable finite-size effects, i.e. a much higher nucleation rate, are absent for systems with more than 1000 atoms [58], although some discrepancy could occur in systems of up to a million atoms [59], particularly at low undercooling.

\subsection{DPS methodology}

Discrete pathways were found by connecting successive minima in the trajectory using the doubly-nudged [60] elastic band [61] approach for double-ended searches to locate transition state candidates. These candidates were subsequently refined using hybrid eigenvector-following [38, 39,61]. This approach, implemented in the OPTIM program [62], finds a pathway between two successive minima in the quenched trajectory, which can involve a single transition state or a series of transition states and intervening minima. The limited-memory Broyden-Fletcher-Goldfarb-Shanno (LBFGS) algorithm of Liu and Nocedal $[63,64]$ was used for the local minimisations. An optimisation was deemed to have converged when the root-mean-square gradient fell below $10^{-6}$ reduced units. The PATHSAMPLE program [65] is a driver for OPTIM, directing the construction and refinement of a stationary point database formed from the connected discrete paths. 


\subsection{Permutational alignment}

Alignment of endpoints is critically important to obtain pathways via geometry optimisation [66]. The alignment, minimising the Euclidean distance in $3 N$ dimensions, of $N$ distinguishable atoms in two different configurations is easily achieved using a quaternion procedure [67]. However, there is no longer a deterministic solution to this problem when atoms are indistinguishable, i.e. they can be permuted. A sequence of steps is necessary. Firstly, the Euclidean distance metric is minimised for each set of permutable atoms. For a fixed centre of coordinates and a fixed orientation, the distance between two configurations can be minimised using a shortest augmenting path algorithm [68]. This minimisation is then followed by overall translational and rotational alignment. The sequence of permutational and translational/orientational alignment can produce a different result depending on the initial orientation. To find the global minimum for the Euclidean distance it may be necessary to start from a number of random initial orientations. For a system with periodic boundary conditions, such as the bulk Lennard-Jones model considered here, arbitrary overall rotations are not allowed, but cell symmetries must be considered. The alignment process described above was repeated for configurations corresponding to all 48 symmetry operations of the octahedral supercell to find the smallest distance between two minima. It is important to note that for any condensed phase described by a periodic supercell, there is no well-defined centre of coordinates. The resulting lowest value for the Euclidean distance is for a given centre of coordinates. However, for aligning structures to obtain pathways, it is often sufficient to use the atoms in the primary supercell to define the centre of coordinates.

It is important to recognise a previously-encountered structure and it is also necessary to recognise permutation-inversion isomers whenever they are encountered. For bulk systems, the identification of identical structures and isomers requires a different procedure and is separate from any alignment involving an arbitrary 'centre of coordinates'. An attempt is made to overlay the structures by first translating one of them to align a particular pair of atoms, one from each configuration. We then compare the positions of the other atoms in the two structures, calculating the shortest distances between pairs. Equivalent isomers are identified by a 'zero' overall distance within a given tolerance. As soon as one atom cannot be matched in the other structure and the overall distance between the two structures is larger than the 'zero' threshold, the test can be aborted. The test is then repeated for all possible initial partners, and it is sufficient to compare every possible partner in one configuration with a single atom in the second configuration. The whole process was also repeated for all symmetry operations of the cubic box. Using this procedure we find that matching identical structures or permutational isomers is an efficient and robust process.

The above alignment procedures may seem onerous but are generally less computationally expensive than operations that involve evaluating the potential, and they can greatly reduce the computational expense of finding connected pathways, especially for large systems with many equivalent atoms.

\subsection{Disconnectivity graphs}


For an $\mathrm{N}$-atom system, the potential energy is described by a $3 \mathrm{~N}$-dimensional function, which is hard to visualise. A disconnectivity graph analysis focuses on the local minima and the transition states that connect them [69, 70]. Disconnectivity graphs are tree graphs, and we describe different patterns of organisation informally by analogy. In particular, a 'palm-tree' structure corresponds to a landscape with a well-defined global minimum, which can be reached by traversing relatively low downhill barriers between successive minima where there are relatively large systematic changes in potential energy [70]. Palm tree landscapes represent a universal form for 'structure-seeking' or 'self-organising' systems [71]. Protein folding, 'magic numbers' in mass spectra, selfassembly, and crystallisation can generally be attributed to a 'palm tree', funnelled landscape, where a few low energy structures can be located from an exponentially large number of alternatives. We expect nucleation and growth in a crystal to be characterised by such a landscape.

Disconnectivity graphs show the energy of each individual minimum, and also include information covering the connections between minima via the highest transition state on the lowest energy pathway between two minima. The detail of the full pathway, and hence the mechanism for a transition, is not shown directly, but is contained in the underlying database.

\section{Results}

In the following sections, we will describe the energy landscape obtained from discrete path sampling for the 864-atom Lennard-Jones supercell defined above. Having found an initial path between the liquid and crystalline states we then explored the landscape in the crystal/nucleation region around it. In section 2.1 we will use order parameters to examine the resulting database of structures, and we describe in detail the configurations found in the low potential energy region in section 2.2. We will discuss further refinement of the database after the formation of an initial path in section 2.3, and compare our results to previous studies of crystallisation in Lennard-Jones solids in section 2.4. In section 2.5 we analyse a particular nucleation trajectory in more detail, examining the path that makes the largest kinetic contribution in our database of structures.

Figure 1 shows the database formed once an initial path has been found between two well-separated states, one obtained by minimisation from the well-equilibrated liquid phase, the other the FCC crystal. This initial search was followed by some limited refinement to remove high barriers between the lowest-energy minima, using the FREEPAIRS method implemented in PATHSAMPLE [65]. In this procedure, pairs of minima are selected for connection attempts based on the ratio of the free energy barrier to the free energy difference between the minima [34]. Approximate free energies are calculated using the harmonic normal mode frequencies. In the initial path formation, we did not distinguish periodic-cell isomers, but it was necessary to do so at this point, as a number of permutational isomers appeared in the initial database. There are 34219 minima and 46036 transition states within the connected region of configuration space that we have sampled, and all these stationary points are included in figure 1. 
In total 86071 minima and 76070 transition states are contained within the database, but the remaining stationary points are disconnected. The two endpoint minima are labelled on the disconnectivity graph as 'liquid' and 'FCC'. The low-energy structures are clearly distinguished, and apart from a number of outlying minima, which we will discuss further in section 2.3, the landscape appears to be funnelled towards the low energy structures, as expected for efficient crystallisation. There is also only one main funnel, suggesting that there is little competition between structures until the lowenergy region is reached.

\subsection{Order parameters}

In constructing the initial discrete path between endpoints and expanding the database of minima and transition states, we have tried not to bias the simulation in any way. There is no order parameter or preconceived reaction coordinate. Our initial guess for every connection attempt between minima uses the shortest Euclidean distance, i.e. the shortest distance that each atom has to move to get from one structure to another. The connection attempts start with linear interpolation and refinement of this interpolation to produce a connected series of minima and transition states generally moves the configurations a long distance from the initial straight line path. No additional structural information was used in the search for pathways. However, having formed an initial pathway and a database of structures, we can check the sampling and hence, the validity of the methods used, by analysing the structures.

The local environment around each individual atom can be classified as liquid or crystalline by first examining the location of the particle's neighbours. A bond orientational order parameter $q_{l m}$ is defined for each particle [72], using spherical harmonics and the $N_{b}$ neighbours within a radial distance $r_{c}$ :

$$
q_{l m}=\frac{1}{N_{b}} \sum_{j=1}^{N_{b}} Y_{l m}\left(\theta_{i j}, \phi_{i j}\right) .
$$

$q_{l m}$ describes the local environment around each individual particle. Crystallinity can be identified by a long-range repetition of this local order and hence, the next step is to determine the similarity of the environments around two neighbouring particles. This similarity can be quantified by taking the normalised scalar product of a chosen vector, $q_{6 m}$, with a cutoff, $d_{c}$, to determine if the particles have the same structural signature. A particle is then identified as crystalline if the number of structural connections is larger than a cutoff value, $n_{c}$. We define the values of these cutoffs as follows: $r_{c}=1.3909, d_{c}=0.6$ and $n_{c}=7$. This method can be used to identify crystalline clusters with varying shapes and sizes, and to determine the size of the largest solid-like cluster (or crystallite), $N_{\text {clust }}$, in each configuration [73]. In figure 1, each minimum is colour-coded according to the value of $N_{\text {clust }}$. A clear distinction can be seen between the liquid regions ( $N_{\text {clust }}<50$, red $\Rightarrow$ orange) at high potential energy and the crystalline regions ( $N_{\text {clust }}>800$, blue) at low potential energy. $N_{\text {clust }}$, as defined above, uses standard cutoffs, which are usually applied to dynamical configurations that incorporate 




Figure 1. Disconnectivity graph showing the potential energy landscape for a bulk Lennard-Jones system of 864 atoms with periodic boundary conditions. Discrete path sampling was used to construct an initial path between a liquid-like state and an FCC crystal. The two endpoints are labelled and the graph shows all the connected minima and transition states found in the formation of a complete path. The minima displayed in the graph are coloured according to the size of the largest crystalline cluster, $N_{\text {clust }}$. Fully crystalline structures with $N_{\text {clust }}=864$ are shown in blue and structures with no atoms in crystalline environments are shown in red.

thermal noise. For potential energy minima, these criteria are looser than necessary, and hence many structures appear to be perfectly crystalline.

The size of the largest crystalline cluster has been used as an order parameter to drive simulations of nucleation, for example in transition interface path sampling [50] and forward-flux sampling [48]. Several studies have suggested that a combination of the nucleus size and the degree of crystallinity of the nucleus may be a better order parameter [49, 74], but measures of crystallinity can perform differently under different conditions of temperature and pressure [75]. Local crystallinity has also been combined with the potential energy of the system as a second reaction coordinate [76]. In view of this debate and our large sample of potential energy minima, we show the relationship between the potential energy of a particular configuration and the size of the largest crystalline cluster contained within the configuration in figure 2 . There is a clear correlation between these two parameters, and at least for the Lennard-Jones system, $N_{\text {clust }}$ 
The potential energy landscape for crystallisation of a Lennard-Jones fluid

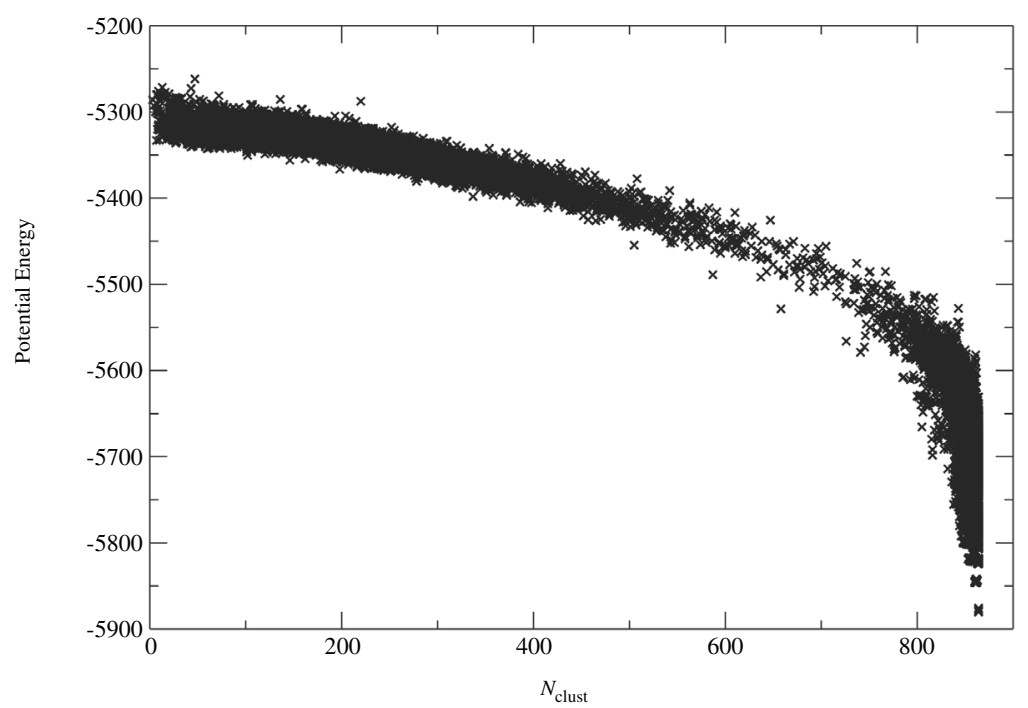

Figure 2. Potential energy as a function of the size of the largest crystalline cluster ( $N_{\text {clust }}$ ) for local minima of the $\mathrm{LJ}_{864}$ system sampled between liquid and crystalline states.

does indeed appear to be a reasonable order parameter, driving the system towards lower potential energy. The influence of the finite box size, 864 atoms, is also evident in this figure. There is a rapid drop in potential energy for crystal structures that fit precisely into the periodic box.

\subsection{Low-energy structures}

Bond orientational order parameters averaged over a particle and its nearest neighbours can identify locally crystalline structures as FCC, HCP, BCC or icosahedral. The crystal packing around each individual particle can be characterised according to the stacking of neighbouring atoms around the central particle. The $q_{l m}$ vectors for each particle are replaced by an average over this atom and its nearest neighbours. The averaging narrows the distribution of order parameters, and a distinct combination of third order invariants $w_{4}$ and $w_{6}$ of the averaged complex vectors $\bar{q}_{l m}$ can be found for each type of stacking [77], see table 1. FCC and HCP lattices are well defined by this scheme, but the $w_{4}$ distribution for a BCC structure is broad [50]. An İ̄3d phase recently discovered for the LJ system [44] may form part of the BCC distribution and roughly falls into the uncharacterised region of the $w_{4} / w_{6}$ parameter space. Such structures have previously been referred to as 'else' [50] and will be labelled here as BCC2.

Figure 3 focuses on the bottom of the potential energy landscape and four distinct low-energy crystals with very similar potential energies can be identified. In this figure, the graph is coloured according to the degree of HCP order, i.e. the number of particles within the configuration surrounded by an HCP environment. Minima with fully HCPordered configurations are blue, and minima with no HCP order, which in this case are in fact FCC-ordered, are shown in red. The four perfect crystals (illustrated at the bottom of figure 3 ) are pure FCC, pure HCP, and two combinations of FCC and HCP stacking: $(\mathrm{FCC}+2 \mathrm{HCP}) / 3$ and $(2 \mathrm{FCC}+\mathrm{HCP}) / 3$. In fact, these are the only possible stacking combinations that fit precisely into the periodic box. Finding all four perfect 
The potential energy landscape for crystallisation of a Lennard-Jones fluid

Table 1. Third order invariants $w_{4}$ and $w_{6}$ are constructed from averaged local order parameters [77].

\begin{tabular}{lcc}
\hline & $w_{4}$ & \multicolumn{1}{l}{$w_{6}$} \\
\hline fcc & -0.15932 & -0.01316 \\
hcp & 0.13410 & -0.01244 \\
bcc & 0.15932 & 0.01316 \\
icos & - & -0.16975 \\
\hline
\end{tabular}

Note: In the ideal case a combination of the two values can be used to distinguish between all the relevant structures.

crystals, despite starting with only the FCC crystal as an endpoint in the simulation, provides some confidence that the sampling has been effective.

Another well-defined funnel is visible on the left of figure 3, at slightly higher potential energy. Structures within this funnel also contain HCP and FCC layers, but have a stacking combination that does not percolate correctly across the periodic boundary and hence has slightly imperfect stacking. The structure found at the bottom of this funnel is also shown in figure 3. A layered cluster of atoms (green) in different environments, identified by the order parameter as $\mathrm{BCC}$ or $\mathrm{BCC} 2$, is required to compensate for the stacking error.

Each perfect crystal is found at the bottom of a funnel containing many other very similar minima with slightly imperfect structures. These minima differ only in a few shorter and longer interparticle distances. These are not visible defects, but imperfections can be identified if very strict order parameters are used.

Relatively low-energy pathways exist between the pure FCC and pure HCP crystals. In fact, due to the finite box size, these two structures can interconvert by the sliding of four adjacent planes of atoms, two in one direction and two in the opposite direction. This sliding mechanism is exactly the process chosen in [78] as a Monte Carlo move to interconvert the two structures. As FCC-HCP interconversions are possible at relatively low potential energy, we would expect to find all rHCP structures within the same funnel in the corresponding disconnectivity graph.

\subsection{Refining the database}

Once an initial pathway between the two endpoints has been discovered, we can focus on refining the resulting database of stationary points. Our goal is to obtain a more complete sample of the configuration space between the liquid and crystalline regions. Figure 1 reveals a significant degree of kinetic frustration in the database. Such frustration is not unusual after the formation of the initial path. The frustration may well be artificial and can occur when local minima are found during attempts to make discrete paths. These minima are added to the database, but lie behind high barriers where low-barrier paths actually exist but have not yet been identified [34, 36]. Selected connection attempts can be made to remove artificial frustration; minima are selected according to the barrier height divided by the energy difference to the product minima (often the global minimum), analogous to a measure of frustration. This procedure corresponds to the UNTRAP database refinement keyword in the PATHSAMPLE program, as described elsewhere [36, 79]. 

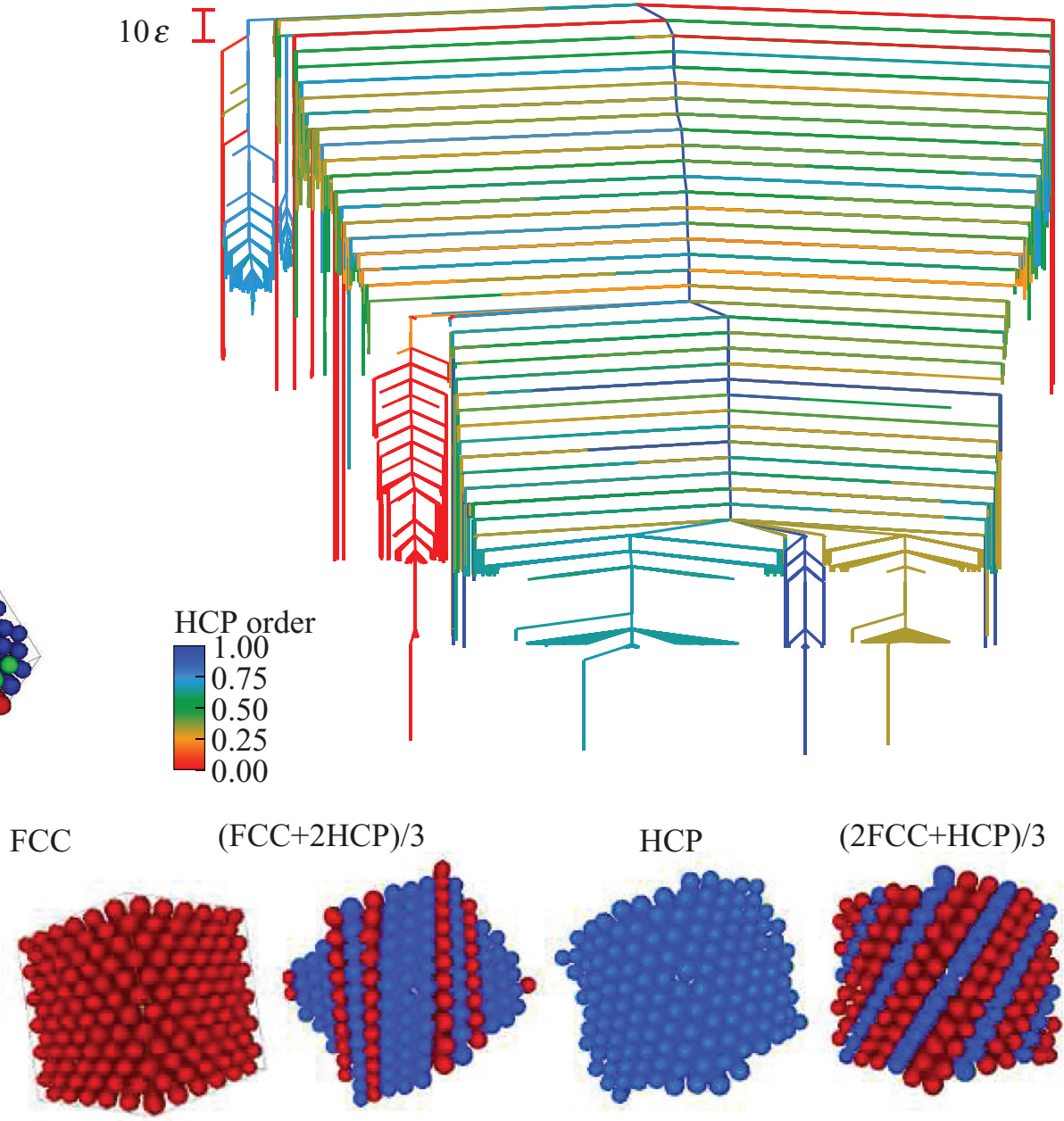

Figure 3. Disconnectivity graph for the low potential energy, crystalline, region of $\mathrm{LJ}_{864}$. The four distinct perfect crystals are shown, as well as an rHCP structure, which does not percolate across the periodic boundary. The minima are coloured according to the number of atoms in HCP environments, with ideal HCP crystals and FCC crystals coloured blue and red, respectively. The structures show atoms in FCC, HCP, BCC and BCC2 environments in red, blue, green and dark green, respectively.

The outlying minima are isolated and not contained within separate funnel structures, which is a good indication of artificial frustration. The high-energy barriers generally connect to the main funnel through the liquid state and, even if they correspond to distinct crystal structures, we would expect interconversions to be possible at potential energies well below the liquid states. In fact, no distinct structures were identified, and all the outlying minima contain HCP and FCC stacking to varying degrees. Hence lower energy barriers to the main funnel must exist.

Running the UNTRAP procedure in PATHSAMPLE [65] connected many of these low-lying minima to the main funnel, and lower energy pathways were easily identified in many cases. However, some problems occurred with the calculation of distances between structures defined under periodic boundary conditions. Firstly, we would like to make a connection attempt, i.e. search for a discrete path, between the outlying 
minimum and the minimum in the main funnel separated by the shortest Euclidean distance from this structure. Unfortunately the distance calculation is computationally expensive when permutations are accounted for, and with around 25000 minima in the main funnel, it becomes time consuming to check them all to find the shortest distance. Secondly, we find a number of seemingly perfect FCC structures that are separated from the pure FCC crystal by very large distances, comparable to the distance between a liquid and crystal structure. Thirdly, crystalline minima with small localised defects are very close in configuration space to the corresponding ideal structure. However, permutational alignment can result in many atoms having to move a small amount between the two structures, rather than just the necessary moves in the defective area.

The first problem can be addressed by characterising the structure of minima in the main funnel, using order parameters such as those described in section 2.1. The distance calculation can then be limited to minima with similar structures to find the most appropriate partner. It is important to note that structural information is only used to select this partner, and there was no structural bias in the search for a discrete path between minima. A new keyword for this method, METRICUPAIR, has been implemented in PATHSAMPLE [65].

Some seemingly ideal FCC structures do not have the same energy as the perfect FCC structure in the main funnel and hence, imperfections must exist. Tightening the three cutoff criteria in the calculation of $N_{\text {clust }}$ reveals small regions of imperfect crystalline environments. Examining the structures shows that these imperfections arise because the FCC lattice is not commensurate with the periodic box. The FCC stacking cannot percolate correctly, and some slightly smaller and other slightly larger lattice spacings are required, leading to imperfections. Once recognised, it is easy to characterise these structures by the angle of the lattice relative to the periodic box, and this angle can then be used as an additional order parameter for choosing a partner minimum in the main funnel. Figure 4 shows an example of such a 'shifted' FCC structure, where a non-zero angle between the stacking planes and the periodic box outline is clearly visible. We find structures at a whole range of angles to the periodic box, but the most common examples correspond to angles of $0^{\circ}$ and values below $7^{\circ}$, at small angles to the periodic box. It seems likely that such structures will appear whenever bulk systems are represented using periodic boundary conditions. However, they do not appear to have been discussed before, to the best of our knowledge.

In the third case, if there is a small defective area, the change in the centre of coordinates can cause a misalignment of the two structures. All three problems also highlight an issue with the Euclidean distance method, particularly when used with an arbitrary centre of coordinates, which arises with periodic boundary conditions. The shortest distance between two structures may not bear much relation to the most kinetically relevant pathway. If we aim to sample the landscape widely, rather than simply making a direct connection between two structures, this guess for the initial interpolation avoids bias and is a reasonable choice. However, when we are removing artificial frustration and looking for connections between low-energy crystal structures it is no longer necessary to sample in this way and we need a better method for permutational alignment.

It would be possible to extend the method described in section 1.6, which is currently used to test for exact matches and isomers, to calculate distances for every possible 




Figure 4. An FCC structure with layers stacked at an angle to the periodic box (outlined in grey).

alignment of two pairs of atoms, one from each structure. Aligning atoms rather than the centre of coordinates is particularly appropriate for crystalline structures, as any exactly matching crystalline regions are unlikely to change on the pathway from one structure to another. However, the method was previously used to find an exact match. If instead, we want to find the closest match to another structure, we can no longer abort the test as soon as one atom does not match, and the process becomes computationally prohibitive.

Instead, we have adopted a compromise approach, focusing on finding a short distance between structures rather than the shortest possible distance. The atom matching method proceeds as described in section 1.6 for exact matches, but a test does not abort when one atom does not match. Instead, a count of the number of atomic matches is maintained as the process cycles over different pairs of atoms and different box orientations. If any alignment has fewer matches than the current maximum the test is immediately aborted. Also, if several different pairs of atoms have been aligned in one orientation, but no improvement is found in the number of matches, the process jumps to the next orientation. In this way, we can find permutational alignments where crystalline regions are matched without costly distance calculations. A new keyword, ATOMMATCHDIST, has been implemented in the PATHSAMPLE and OPTIM programs for this procedure $[62,65]$. Once the initial alignment has been found, it is not necessary to realign structures during the connection attempt and the search proceeds much more efficiently, with no further need to check for the presence of permutational isomers.

Figure 5 shows the resulting potential energy landscape after much of the artificial frustration has been removed. There are now 142069 connected potential energy minima and approximately 300000 minima in the database. Structures that contain at least $67 \%$ FCC stacking are labelled according to a colour scale, which shows the angle of the stacking to the periodic box. A lattice angle of zero (red) indicates a lattice that is commensurate with the box. Structures with angles between zero to $45^{\circ}$ are present in the database, but only the smaller, more commonly occurring angles are selected in the visualisation. 


\subsection{Growth of crystalline clusters}

In figure 6 , we examine the average composition of the largest crystalline cluster as a function of the cluster size for comparison with previous results [50]. Cluster sizes between 1 and 200 are shown, corresponding to the region where a critical nucleus is forming and where most of the structures in our database can be found. The top panel shows the structural composition of the entire largest cluster. Our initial path was constructed between a minimum quenched from the equilibrium liquid and a perfect FCC crystal. However, the average composition of the cluster, in terms of the relative proportion of FCC and $\mathrm{HCP}$, is in good agreement with [50]. As the cluster size grows to 100 atoms, FCC stacking becomes increasingly favourable, and a corresponding decrease is seen in the degree of HCP packing. Extrapolation of the results would suggest that this discrepancy grows from an original $1: 1$ distribution. The proportion of $\mathrm{BCC}$ and $\mathrm{BCC} 2$ atoms remains fairly constant at 0.2 for all cluster sizes, with an equal contribution from atoms in the two environments.

The proportion of FCC atoms is higher than the value reported for the overall cluster in previous work [50], and actually more closely resembles the results obtained previously for the cluster core. The bottom figure shows our results for the core, i.e. nonsurface particles. The cluster cores are even more strongly FCC dominated, with $80 \%$ of particles in an FCC environment. There are several possible explanations for this difference, including a systematic bias, and further simulations may be necessary to ensure that we have adequately sampled routes to other crystal structures. Another possibility is that atoms strongly affected by thermal noise, such as those on the surface of the cluster, are identified as BCC in real-space trajectories, but when the instantaneous configuration is quenched to a local minimum the particles relax to more stable FCC environments. As atoms are surrounded by further crystalline environments, their positions become more well-defined, and their environments are now recognised as FCC. In simulations of soft [80] and hard [81] colloids, crystal nuclei have been found to exist within a cloud of pre-ordered surface particles that are highly correlated with their nearest neighbours but not within a crystal structure. Boundaries are fuzzy and it is unsurprising that the surface of a crystalline cluster should be ill-defined and very dependent on the chosen parameters.

\subsection{Pathways}

For every minimum in the database, we calculate a mean waiting time for any transition to occur using the harmonic normal mode approximation and transition state theory [17]. There are a number of possible transitions out of an individual minimum and we can associate a branching probability with each one. Using this information we can evaluate phenomenological rate constants for the overall transition and also determine the contribution made to these rate constants by a particular discrete path [15-17]. The discrete path that makes the largest contribution to the rate constant when intervening minima are treated in the steady state approximation can be extracted from a DPS database using a network formulation [33] and Dijkstra's shortest-path algorithm [41]. We refer to such discrete paths as the 'fastest' paths, noting that the analysis also 


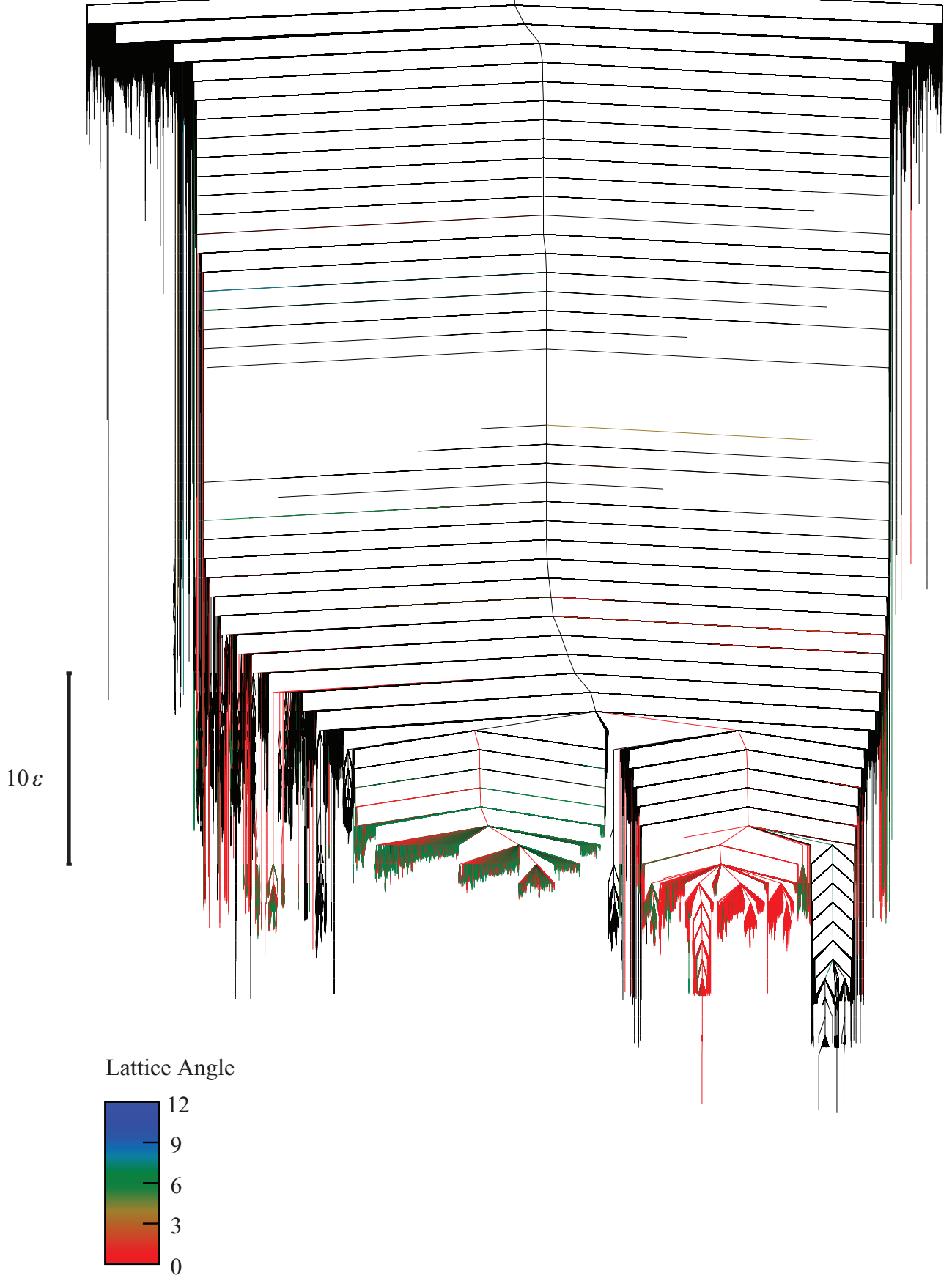

Figure 5. Disconnectivity graph showing the potential energy landscape of the $\mathrm{LJ}_{864}$ system. Geometry optimisation was employed within the discrete path sampling framework to obtain an initial discrete path between the liquid and a FCC crystal. The resulting database of minima and transition states was then systematically refined to remove most of the artificial frustration. Minima with at least $67 \%$ atoms in FCC environments are coloured according to the angle of the FCC lattice relative to the periodic box. The other minima are shown in black. 
The potential energy landscape for crystallisation of a Lennard-Jones fluid
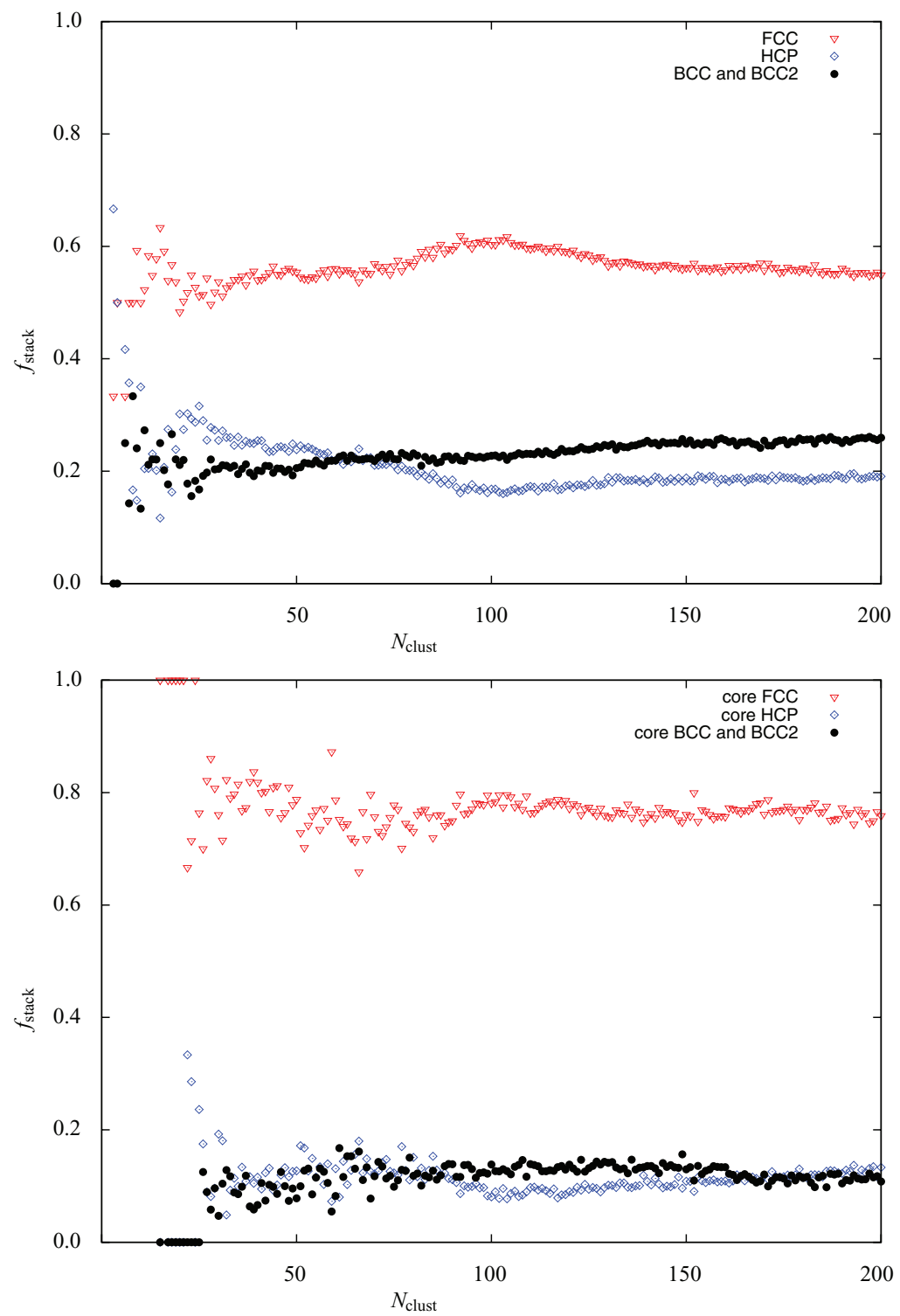

Figure 6. The average proportion of particles located in FCC, HCP and, BCC and $\mathrm{BCC} 2$ environments plotted as a function of the size of the largest crystalline cluster, for both the whole cluster and the cluster core. The figures show cluster sizes from 1 to 200, the region where a critical nucleus is forming.

includes a factor corresponding to the conditional occupation probability of the starting minimum [15-17].

The 'fastest' paths are useful as a focus for further landscape exploration. They also provide individual trajectories, with known kinetic importance, for analysis. Here we will examine the evolution of the 'fastest' path from the initial liquid state to a perfect crystal, extracted from our current database. Figure 7 shows the size of the largest crystalline cluster, i.e. the order parameter $N_{\text {clust }}$, plotted against individual steps (minima) on the discrete path. We observe the formation of a critical nucleus with approximately 100 atoms before growth rapidly propagates across the periodic boundary. Figure 8 shows the last finite crystalline cluster. The FCC stacking at the core of the cluster is clearly identified, and there is some HCP stacking as well as a 
number of BCC and BCC2 atoms at the cluster surface. To obtain the 'fastest' path, we have directed the system to start from the liquid and form a perfect crystal, rather than forming any crystal structure. We see an interesting effect as a slightly defective crystal forms first and then the defects anneal out to form a perfect $(2 \mathrm{FCC}+\mathrm{HCP}) / 3$ crystal.

Figure 9 is a disconnectivity graph based on the 'fastest' path. The graph is coloured according to $N_{\text {clust }}$ and the formation of the critical nucleus occurs within the orange region of the graph. A rapid drop in potential energy follows as the system crystallises and growth occurs across the periodic boundary. Such rapid drops have been seen in other systems [82].

\subsection{Binary Lennard-Jones}

There has been recent interest in studying the interplay between glass formation and crystallisation [83-85]. Binary Lennard-Jones (BLJ) mixtures, which are good glassformers and do not crystallise easily, are good candidates for such a study. Of the two most popular models, the Wahnström model [86] crystallises more easily [87] than the Kob-Andersen model [88]. For a model similar to the Wahnström model, nucleation has been studied and compared with the single-component fluid [50].

Here, using the techniques developed for the pure Lennard-Jones fluid described above, we sample the potential energy landscape between the supercooled liquid and crystal for a 60-atom BLJ mixture of the Kob-Andersen variety. We start from a sample of the potential energy landscape previously obtained [56] for the supercooled liquid at a temperature of 0.76 and use DPS to connect the supercooled regime to the lowest-energy crystal [89]. The resulting landscape is shown in a disconnectivity graph in figure 10, with minima coloured according to the size of the largest crystalline clust, $N_{\text {clust }}$. As expected, $N_{\text {clust }}$ is not a natural order parameter for this system and one advantage of our method is that no order parameter is used to drive the simulation.

\section{Conclusions}

We have sampled the potential energy landscape of a bulk Lennard-Jones system, exploring the configuration space between the fluid and crystalline states. Starting from a single liquid and a single crystalline minimum, we have used geometry optimisation within the discrete path sampling framework to create an initial path between these two states, without the use of any reaction coordinate, and without requiring any structural analysis to guide the simulation. Hence we have demonstrated the successful application of discrete path sampling to bulk systems. We also explore the configuration space between supercooled liquid and crystal for a good glass-forming binary Lennard-Jones system, and provide a visualisation of the potential energy landscape.

For the single-component Lennard-Jones potential, we also study the effect of the periodic box on the crystal structures formed. The initial exploration of the potential energy landscape identified four perfect crystal structures, as well as a large number 
The potential energy landscape for crystallisation of a Lennard-Jones fluid

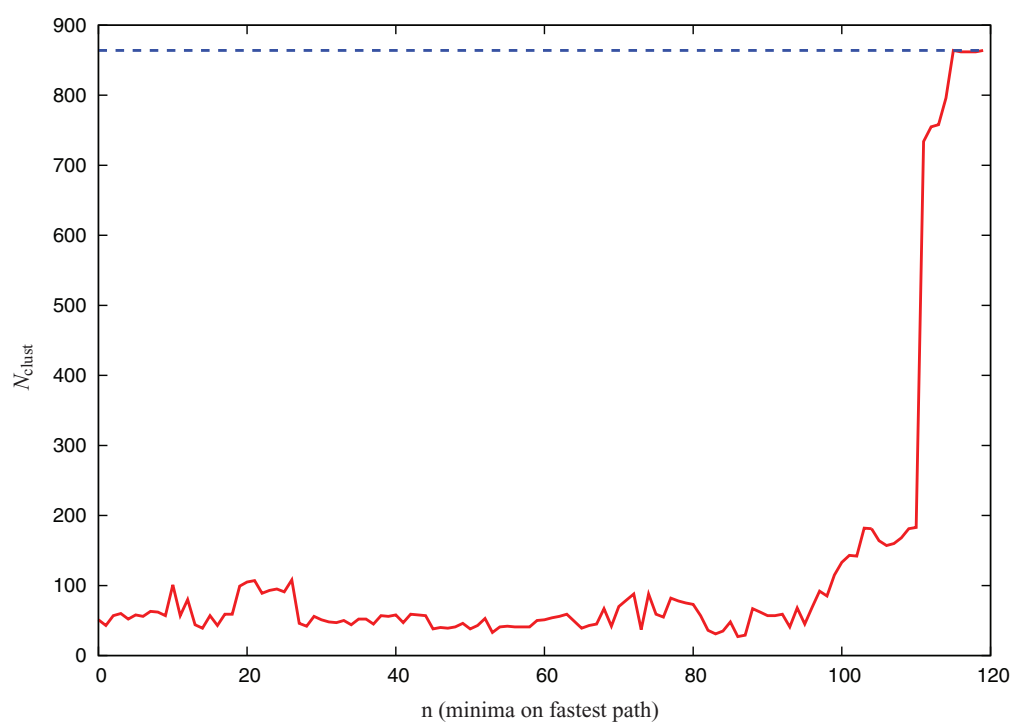

Figure 7. The size of the largest crystalline cluster, the order parameter $N_{\text {clust }}$, is illustrated for individual minima on the 'fastest' discrete path.

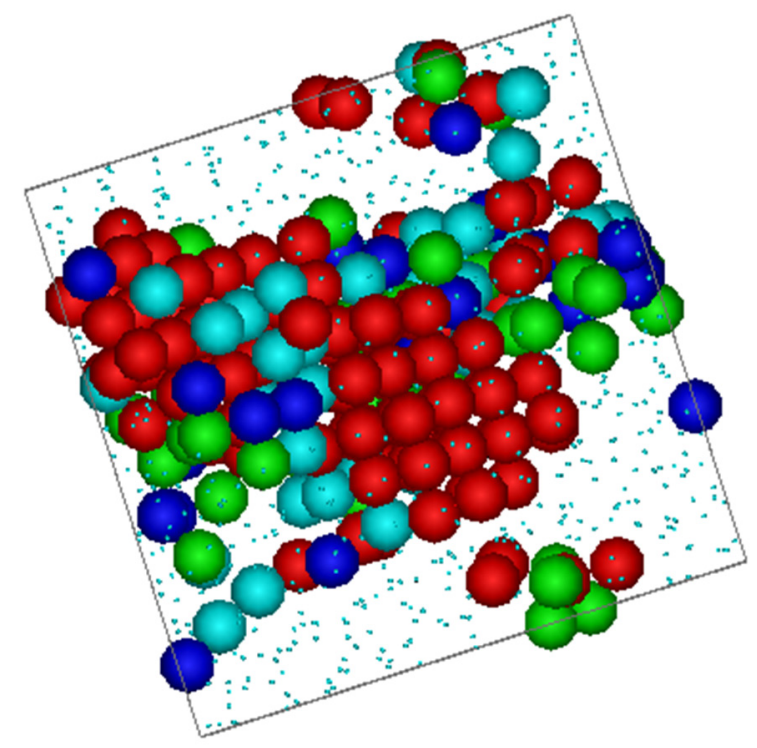

Figure 8. The last crystalline cluster on the 'fastest' path, before a rapid growth process forms a fully crystalline structure. Atoms in crystalline environments are shown by large coloured spheres. Atoms surrounded by FCC, HCP, BCC and BCC2 stacking are shown in red, green, dark blue and light blue, respectively. Atoms in liquid-like environments are indicated by small blue dots.

of defective crystals. There are certain combinations of HCP and FCC layers (rHCP configurations) that do not percolate the periodic box. We also identified FCC packings that appear perfect, but involve small changes in the internuclear distances because the stacking is not commensurate with the periodic box. 


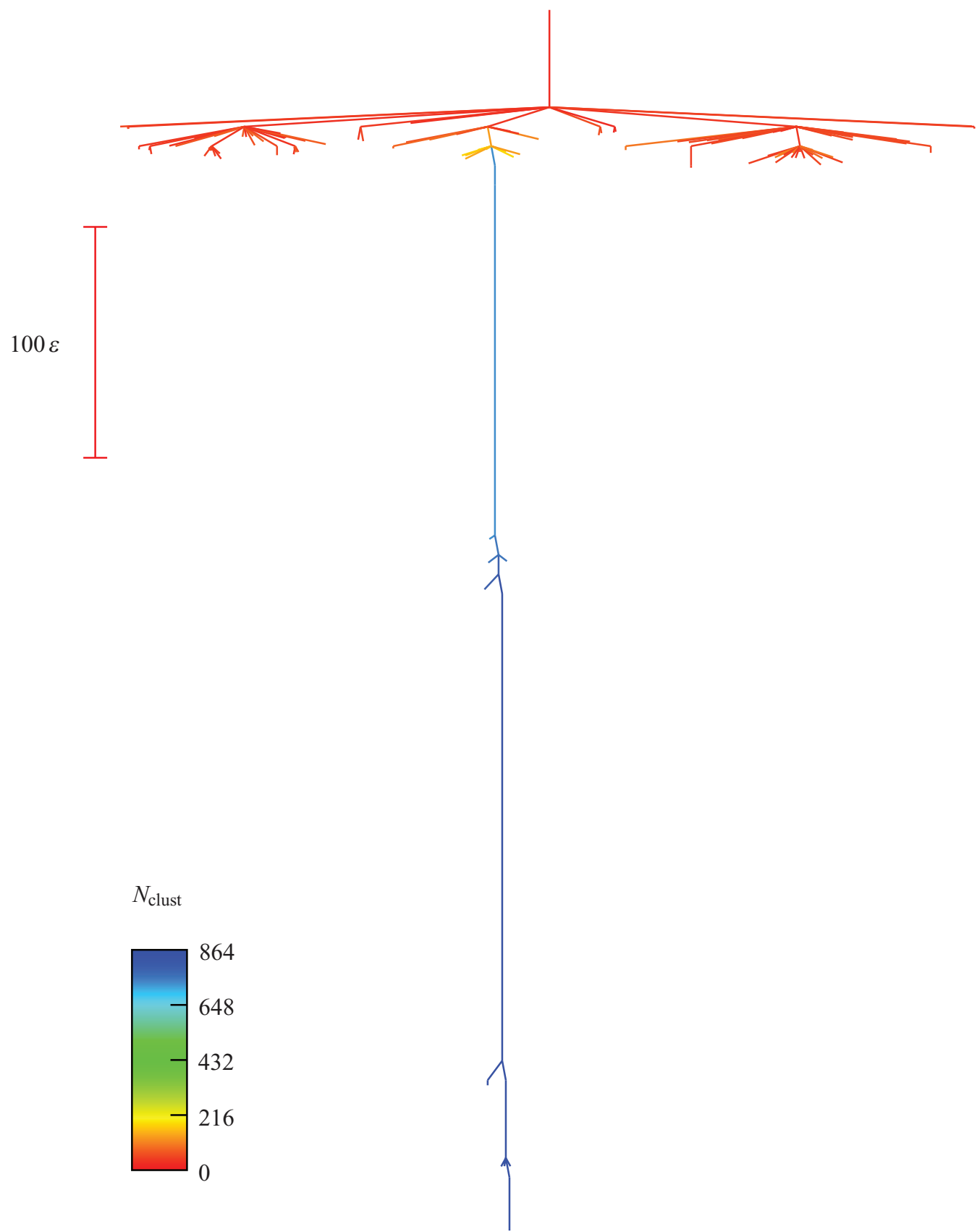

Figure 9. Disconnectivity graph showing the relative potential energy of minima on the 'fastest' path and the connections between these minima. The colour scale shows the size of the largest crystalline cluster within each minimum. The lowest energy minimum is a perfect $(2 \mathrm{FCC}+\mathrm{HCP}) / 3$ crystal.

\section{Acknowledgments}

We acknowledge the Engineering and Physical Sciences Research Council, UK (EPSRC) for funding under Programme Grant EP/I001352/1. Additional data related to this publication is available at the University of Cambridge data repository (www.repository. 
The potential energy landscape for crystallisation of a Lennard-Jones fluid

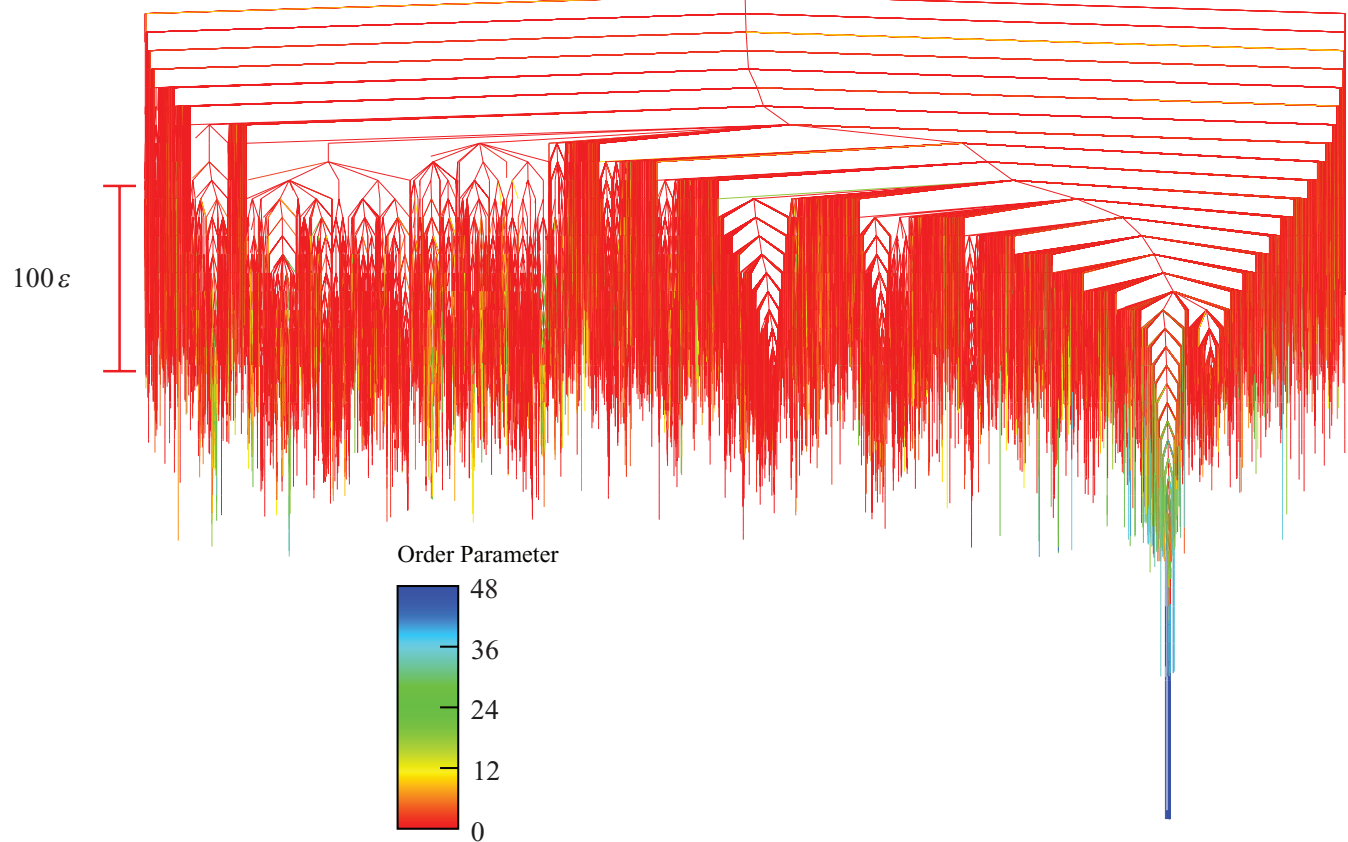

Figure 10. Disconnectivity graph showing the potential energy landscape for a bulk Binary Lennard-Jones system of 60 atoms with periodic boundary conditions. Discrete path sampling was used to construct an initial path between a supercooled liquid state and the lowest-energy crystal. The graph shows all the connected minima and transition states found in the formation of a complete path. The minima displayed in the graph are coloured according to the size of the largest crystalline cluster of the majority A atoms, $N_{\text {clust }}$. Fully crystalline structures with $N_{\text {clust }}=48$ for the $\mathrm{A}$ atoms are shown in blue and structures with no atoms in crystalline environments are shown in red.

cam.ac.uk/handle/1810/254761). VKD is grateful to Darwin College, Cambridge for a Research Fellowship.

\section{References}

[1] van Blaaderen A, Imhof A, Hage W and Vrij A 1992 Langmuir 81514

[2] Gasser U, Weeks E R, Schofield A, Pusey P N and Weitz D A 2001 Science 292258

[3] Voter A F 1998 Phys. Rev. B 57 R13985

[4] Torrie G M and Valleau J P 1974 Chem. Phys. Lett. 28578

[5] Hamelberg D, Mongan J and McCammon J A 2004 J. Chem. Phys. 12011919

[6] Laio A and Parrinello M 2002 Proc. Natl Acad. Sci. USA 9912562

[7] Bussi G, Gervasio F L, Laio A and Parrinello M 2006 J. Am. Chem. Soc. 12813435

[8] Sørensen M R and Voter A F 2000 J. Chem. Phys. 1129599

[9] Dellago C, Bolhuis P G, Csajka F S and Chandler D 1998 J. Chem. Phys. 1081964

[10] Dellago C, Bolhuis P G and Chandler D 1999 J. Chem. Phys. 1106617

[11] Bolhuis P G, Chandler D, Dellago C and Geissler P L 2002 Annu. Rev. Phys. Chem. 53291

[12] Allen R J, Warren P B and ten Wolde P R 2005 Phys. Rev. Lett. 94018104

[13] Allen R J, Frenkel D and ten Wolde P R 2006 J. Chem. Phys. 124024102

[14] Xu L and Henkelman G 2008 J. Chem. Phys. 129114104

[15] Wales D J 2002 Mol. Phys. 1003285

[16] Wales D J 2004 Mol. Phys. 102891 
[17] Wales D J 2006 Int. Rev. Phys. Chem. 25237

[18] Wales D J 2003 Energy Landscapes (Cambridge: Cambridge University Press)

[19] Murrell J N and Laidler K J 1968 Trans. Faraday Soc. 64371

[20] Wales D J, Doye J P K, Miller M A, Mortenson P N and Walsh T R 2007 Adv. Chem. Phys. 1151

[21] Wales D J and Doye J P K 2003 J. Chem. Phys. 11912409

[22] Stillinger F H and Weber T A 1984 Science 225983

[23] Wales D J 1993 Mol. Phys. 78151

[24] Stillinger F H 1995 Science 2671935

[25] Strodel B and Wales D J 2008 Chem. Phys. Lett. 466105

[26] Bogdan T V, Wales D J and Calvo F 2006 J. Chem. Phys. 124044102

[27] Wales D J and Scheraga H A 1999 Science 2851368

[28] Noé F, Krachtus D, Smith J and Fischer S 2006 J. Chem. Theory Comput. 2840

[29] Noé F, Oswald M, Reinelt G, Fischer S and Smith J C 2006 Multiscale Model. Simul. 5393

[30] Noé F and Fischer S 2008 Curr. Opin. Struct. Biol. 18154

[31] Boulougouis G C and Theodorou D N 2007 J. Chem. Phys. 127084903

[32] Evans D A and Wales D J 2003 J. Chem. Phys. 1199947

[33] Evans D A and Wales D J 2004 J. Chem. Phys. 1211080

[34] Carr J M and Wales D J 2008 J. Phys. Chem. B 1128760

[35] Carr J M and Wales D J 2005 J. Chem. Phys. 123234901

[36] Strodel B, Whittleston C S and Wales D J 2007 J. Am. Chem. Soc. 12916005

[37] Khalili M and Wales D J 2008 J. Phys. Chem. B 1122456

[38] Munro L J and Wales D J 1999 Phys. Rev. B 593969

[39] Kumeda Y, Munro L J and Wales D J 2001 Chem. Phys. Lett. 341185

[40] Carr J M, Trygubenko S A and Wales D J 2005 J. Chem. Phys. 122234903

[41] Dijkstra E W 1959 Numer. Math. 1269

[42] ten Wolde P R, Ruiz-Montero M J and Frenkel D 1995 Phys. Rev. Lett. 752714

[43] Desgranges C and Delhommelle J 2007 Phys. Rev. Lett. 98235502

[44] Eshet H, Bruneval F and Parrinello M 2008 J. Chem. Phys. 129026101

[45] Turci F, Schilling T, Yamani M H and Oettel M 2014 Eur. Phys. J. Spec. Top. 223421

[46] ten Wolde P R, Ruiz-Montero M J and Frenkel D 1996 J. Chem. Phys. 1049932

[47] Desgranges C and Delhommelle J 2007 J. Phys. Chem. B 1111465

[48] van Meel J A, Page A J, Sear R P and Frenkel D 2008 J. Chem. Phys. 129204505

[49] Moroni D, ten Wolde P R and Bolhuis P G 2005 Phys. Rev. Lett. 94235703

[50] Jungblut S and Dellago C 2011 J. Chem. Phys. 134104501

[51] Ostwald W 1897 Z. Phys. Chem. 22289

[52] Woodcock L V 1997 Nature 385141

[53] Heitkam S, Drenckhan W and Fröhlich J 2012 Phys. Rev. Lett. 108148302

[54] Keyes T and Chowdhary J 2001 Phys. Rev. E 64032201

[55] Büchner S and Heuer A 2000 Phys. Rev. Lett. 842168

[56] de Souza V K and Wales D J 2008 J. Chem. Phys. 129164507

[57] Peng L J, Morris J R and Lo Y C 2008 Phys. Rev. B 78012201

[58] Huitema A, van der Eerden J P, Janssen J J M and Human H 2000 Phys. Rev. B 6214690

[59] Peng L J, Morris J R and Aga R S 2010 J. Chem. Phys. 133084505

[60] Trygubenko S A and Wales D J 2004 J. Chem. Phys. 1202082

[61] Henkelman G and Jónsson H 1999 J. Chem. Phys. 1117010

[62] Wales D J OPTIM www-wales.ch.cam.ac.uk/software.html

[63] Liu D and Nocedal J 1989 Math. Program. 45503

[64] Nocedal J 1980 Math. Comput. 35773

[65] Wales D J PATHSAMPLE www-wales.ch.cam.ac.uk/software.html

[66] Wales D J and Carr J M 2012 J. Chem. Theory Comput. 85159

[67] Kearsley S K 1989 Acta Crystallogr. A 45 208-10

[68] Jonker R and Volgenant A 1987 Computing 38 325-40

[69] Becker O M and Karplus M 1997 J. Chem. Phys. 1061495

[70] Wales D J, Miller M A and Walsh T R 1998 Nature 394758

[71] Wales D J 2005 Phil. Trans. R. Soc. A 363357

[72] Steinhardt P J, Nelson D R and Ronchetti M 1983 Phys. Rev. B 28784

[73] Auer S and Frenkel D 2004 J. Chem. Phys. 1203015

[74] Beckham G T and Peters B 2011 J. Phys. Chem. Lett. 21133

[75] Jungblut S, Singraber A and Dellago C 2013 Mol. Phys. 1113527 
[76] Trudu F, Donadio D and Parinello M 2006 Phys. Rev. Lett. 97105701

[77] Lechner W and Dellago C 2008 J. Chem. Phys. 129114707

[78] Jackson A N, Bruce A D and Ackland G J 2002 Phys. Rev. E 65036710

[79] Wales D J and Head-Gordon T 2012 J. Phys. Chem. B 1168394

[80] Lechner W, Dellago C and Bolhuis P G 2011 Phys. Rev. Lett. 106085701

[81] Kawasaki T and Tanaka H 2010 Proc. Natl Acad. Sci. USA 10714036

[82] Xiao P, Sheppard D, Rogal J and Henkelman G 2014 J. Chem. Phys. 140174104

[83] Wang M, Zhang K, Li Z, Liu Y, Schroers J, Shattuck M D and O’Hern C S 2015 Phys. Rev. E 91032309

[84] Banerjee A, Chakrabarty S and Bhattacharyya S M 2013 J. Chem. Phys. 139104501

[85] Royall C P and Williams S R 2015 Phys. Rep. 5601

[86] Wahnström G 1991 Phys. Rev. A 443752

[87] Pedersen U R, Schroder T B, Dyre J C and Harrowell P 2010 Phys. Rev. Lett. 104105701

[88] Kob W and Andersen H C 1995 Phys. Rev. E 514626

[89] Middleton T F, Hernández-Rojas J, Mortenson P N and Wales D J 2001 Phys. Rev. B 64184201 